\title{
Four-Wave Mixing and Generation of Terahertz Radiation in an Alternating-Strain Coupled Quantum-Well Structure
}

\author{
Roberto Paiella and Kerry J. Vahala, Member, IEEE
}

\begin{abstract}
We propose a scheme for exciting steady-state tunneling oscillations of an electronic wave packet in a semiconductor coupled quantum-well structure with strain of the opposite polarity in the two wells. A detailed study of the four-wave mixing process in this structure is then presented, based on the density matrix formalism. Our results show that a resonance peak is introduced in the four-wave mixing frequency response at the tunneling frequency, leading to a significant enhancement in the wavelength conversion efficiency for low temperature operation. Furthermore, we consider this structure under the same excitation condition as a potential source of coherent radiation in the terahertz frequency band.
\end{abstract}

\section{INTRODUCTION}

$\mathbf{T}$ HE study of nondegenerate four-wave mixing (NDFWM) processes in semiconductor optical amplifiers is of considerable importance from both a fundamental and a practical point of view. It has emerged in recent years as a powerful technique for investigating the ultrafast dynamics of excited carriers in semiconductor gain media [1]-[5]. Furthermore, NDFWM in semiconductor traveling-wave amplifiers is a promising candidate for application to wavelength conversion [6], [7] in future broadband multichannel lightwave systems. Its intrinsic advantages in this respect include suitability to high-speed operation and to continuous tuning of the input and output wavelengths, as well as transparency to the modulation format. In addition, FWM can be utilized for fiber-dispersioncompensation by mid-span spectral inversion [8].

In recent years, the FWM conversion efficiency of bulk and multiple quantum-well amplifiers has been measured for detuning frequencies up to several terahertz [1]-[5]. In general, the physical mechanism responsible for FWM is Bragg scattering from the dynamic gain and index gratings resulting from the beating of the input pump and probe waves. Such gratings are associated with modulation of both carrier density and occupation probability in each (sub)band at the pump-probe detuning frequency. Carrier-density pulsations provide the most efficient scattering mechanism; however, they are limited by the recombination lifetime $\tau_{s}$, typically on the order of $100 \mathrm{ps}$ in semiconductor optical amplifiers, so that the effectiveness of the resulting gratings rapidly

Manuscript received August 28, 1995; revised December 11, 1995. This work was supported by the Office of Naval Research (N00014-91-J-1524) and the National Science Foundation (ECS-9412862).

The authors are with the Department of Applied Physics, Mail Stop 128-95, California Institute of Technology, Pasadena, CA 91125 USA.

Publisher Item Identifier S 0018-9197(96)02556-0.
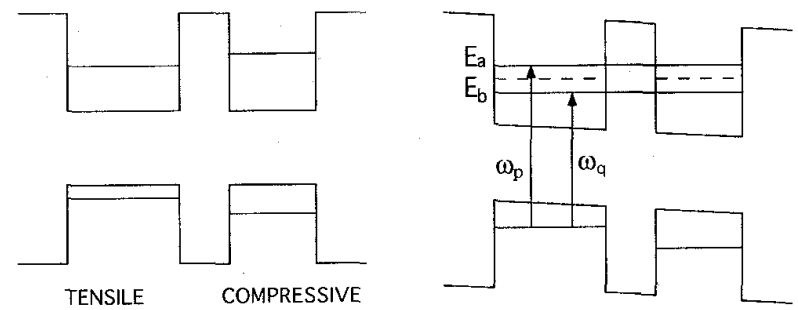

Fig. 1. Schematic band diagram of the alternating-strain coupled quantum-well structure. Under the bias condition of (b), the ground-state levels of the two wells in the conduction band mix to form new bonding and antibonding eigenstates. An electronic wave packet can then be prepared in the tensile-strained (left) well by two TM polarized waves with frequencies as shown.

decreases at detuning rates in excess of a few tens of gigahertz. Modulation of the occupation probability is mediated by intraband processes, which include spectral hole burning [9], arising from the finite time with which a quasiequilibrium Fermi distribution is established in each subband, and carrier heating [10], arising from the finite time with which excited carrier distributions relax to the lattice temperature. Since these processes occur on an ultrafast time scale $(<1 \mathrm{ps})$, they allow for significant NDFWM up to and beyond terahertz detuning rates.

In the present paper, we investigate the NDFWM frequency response of an optical amplifier based on the alternatingstrain coupled quantum-well (ASCQW) structure schematically shown in Fig. 1. In a recent letter [11], we proposed a scheme for exciting in this structure coherent electric-dipole oscillations, which, in turn, introduce a resonance peak in the conversion efficiency at terahertz detuning rates. These results suggested the possibility of tailoring the (third-order) nonlinear optical susceptibility associated with NDFWM in semiconductor optical amplifiers by appropriate design of the amplifier structure. The objective of this paper is to provide a comprehensive account of the theoretical approach used to derive the NDFWM frequency response of the ASCQW structure. Furthermore, we show how the same excitation scheme of such structure can in principle lead to efficient generation of far-infrared radiation through a concomitant second-order wave mixing process.

We start by describing in Section II the basic features of the ASCQW structure and of the proposed excitation scheme, which is based on the dipole transition selection rules in the 
presence of strain. The equations of motion governing the time evolution of the occupation probabilities and dipoles of the various subbands are formulated in the context of the density matrix formalism in Section III and solved in Section IV. The results are then used to discuss the possibility of using this structure to enhance the NDFWM conversion efficiency at terahertz detuning rates (Section V), and as a potential coherent source of submillimeter waves (Section VI).

\section{The Alternating-Strain COUPLED QUANTUM WELl SYSTEM}

A coupled well structure such as that shown in Fig. 1 consists of two quantum wells separated by a barrier thin enough to allow significant overlap of energetically close single-well states. The ground-state levels of the two wells in the conduction band $(|l\rangle$ and $|r\rangle$ for the left and right well, respectively) can be aligned with each other by proper design of the structure and application of a fine-tuning dc field along the growth direction, as illustrated in Fig. 1(b). Then, as a result of state coupling across the heterostructure barrier, $|l\rangle$ and $|r\rangle$ mix to form new stationary states that are delocalized over the two wells. At resonance, these are approximately given by the bonding and antibonding combinations

$$
\begin{aligned}
& |b\rangle=\frac{1}{\sqrt{2}}(|l\rangle+|r\rangle), \quad E_{b}=E_{0}-\frac{\hbar \omega_{t}}{2} \\
& \left.|a\rangle=\frac{1}{\sqrt{2}}(l\rangle-|r\rangle\right), \quad E_{a}=E_{0}+\frac{\hbar \omega_{t}}{2}
\end{aligned}
$$

where $\omega_{t}$ is proportional to the coupling matrix element. On the other hand, for the polarity of the dc bias shown in Fig. 1(b), the valence band levels (both heavy and light holes) in the two wells are sufficiently separated in energy that the holes remain localized in either well.

Since the localized states $|l\rangle$ and $|r\rangle$ are no longer stationary under the bias condition of Fig. 1(b), if an electron is initially prepared in either state, it will then tunnel back and forth through the heterostructure barrier at frequency $\omega_{t}$. The coupled well structure can then be used to set up an electronic wave packet oscillating across the barrier [12], [13], provided one is able to excite a single-well wave packet only. Note that this requires simultaneous excitation of both stationary states $|a\rangle$ and $|b\rangle$. This coherent wave packet dynamic was demonstrated in a series of experiments on (Al)GaAs coupled well structures [14], [15], where the required initial state was prepared by means of ultrashort laser pulses of spectral width larger than $\omega_{t}$ (so as to excite both stationary states) and center frequency tuned to that of the longer-wavelength single-well transition (so as to discriminate between the two wells).

In a NDFWM experiment, the excitation of a single well can be accomplished by introducing strain of the opposite polarity in the two wells, and then appropriately selecting the polarization state of the input pump and probe waves. This technique has been recently used by Zhou $e t$ al. to study interwell transport in alternating-strain multiple quantum-well optical amplifiers [16]. For example, if tensile strain is introduced in the left well and compressive strain in the right well, and the input field consists of two TM polarized waves with frequencies as shown in Fig. 1(b), then, since compressive quantum wells have negligible TM gain, only the linear combination of $|a\rangle$ and $|b\rangle$ corresponding to the single-well state localized in the tensile well $|l\rangle$ will be modulated by the beating of these input waves.

Under these conditions, the occupation probability of both wells behaves like a harmonic oscillator driven at the pumpprobe detuning frequency. A resonance peak is therefore introduced in the NDFWM frequency response at a detuning frequency equal to the natural oscillation frequency $\omega_{t}$ (typically a few terahertz). Furthermore, a steady-state electric dipole oscillation at the driving frequency is established across the conduction band barrier, which acts as a source of submillimeter waves. In the presence of dissipative interactions, such as carrier-carrier and carrier-phonon scattering, the phasecoherent nature of the wave packet oscillatory dynamics is destroyed with a characteristic dephasing time constant $\tau_{d}$. This limits the effectiveness of both processes, and in practice, low temperature operation is required to observe a resonance enhancement in their respective efficiencies. However, the susceptibility responsible for generation of far-infrared radiation is found to be significant, on the order of $10^{-7} \mathrm{~m} / \mathrm{V}$, even at room temperature.

\section{GENERAL FORMALISM}

In this section, we formulate the dynamic equations describing the time evolution of the system's density matrix. For the sake of simplicity, we will treat the case of only one subband in each well. Since the incident optical field couples both the bonding and the antibonding conduction subbands with either valence subband (depending on the field polarization), the ASCQW structure is described as a set of inhomogeneously broadened three-level systems labeled by the two-dimensional wavevector $k$ parallel to the junction plane. It is convenient to write the density matrix $\rho$ corresponding to each such system in the $\{|r\rangle,|l\rangle,|v\rangle\}$ representation, which amounts to replacing each pair of the stationary states $|a\rangle$ and $|b\rangle$ at the given $k$ with their linear combinations given by (1). We consider the case of incident TM radiation, so that $|v\rangle$ here denotes the valence subband in the left well (tensile strained).

Coupling among these basis states includes tunneling across the barrier in the conduction band and dipole interactions between $|l\rangle$ and $|v\rangle$ and between $|l\rangle$ and $|r\rangle$. Taking the origin of the growth axis to be the center of the left well, and neglecting dipole moment matrix elements between states in different wells (in view of their small overlap), the Hamiltonian of the system in this representation is then approximately given by

$$
H=\left[\begin{array}{ccc}
E_{0}^{k}-\mu_{r}^{k} \mathcal{E}(t) & -\frac{\hbar \omega_{t}}{2} & 0 \\
-\frac{\hbar \omega_{t}}{2} & E_{0}^{k} & -\mu_{l v}^{k} \mathcal{E}(t) \\
0 & -\mu_{l v}^{k} \mathcal{E}(t) & E_{v}^{k}
\end{array}\right]
$$

where $\mu$ is the electric dipole moment operator and the superscript $k$ denotes dependence on the in-plane wavevector and will be suppressed in the following. Notice that $\omega_{t}$ is assumed to be independent of $k$, as appropriate if the twodimensional bands $|l\rangle$ and $|r\rangle$ have equal in-plane dispersion relations. 
We consider the case of copropagating pump and probe waves, with the pump much more intense so that only one sideband wave need be considered. The total electric field is then given by

$$
\begin{aligned}
\mathcal{E}(t)= & E_{p} \exp \left(-i \omega_{p} t\right)+E_{q} \exp \left(-i \omega_{q} t\right) \\
& +E_{s} \exp \left(-i \omega_{s} t\right)+\text { c.c. }
\end{aligned}
$$

where $p, q, s$ denote pump, probe and converted signal, respectively, and the detuning frequency is $\Omega=\omega_{p}-\omega_{q}=\omega_{s}-\omega_{p}$.

The damping mechanisms responsible for relaxation of the density matrix include spontaneous recombination and carrier-carrier and carrier-phonon scattering. Spontaneous recombination takes place between valence and conduction band states in the two wells separately, and depletes $|l\rangle$ and $|r\rangle$ while restoring thermodynamic equilibrium at the rate $1 / \tau_{s}$. The effects of the other scattering processes are best formulated in the $\{|a\rangle,|b\rangle,|v\rangle\}$ representation, since they involve relaxation toward quasiequilibrium Fermi distributions, which are properly defined only for the coupled-well energy eigenstates. In this basis, the collisional relaxation rate can be written following the treatment of [17] as (4) shown at the bottom of the page.

Here, the first term in each diagonal element accounts for intraband carrier-carrier scattering, which establishes a quasiequilibrium Fermi distribution $f_{x}$ in each subband $(x=$ $a, b$, or $v$ ) with a time constant $\tau_{1 x}$ and is responsible for spectral hole burning. $f_{x}$ is a function $f_{x}\left(N_{x}, T_{x}\right)$ of the subband carrier density $N_{x}$ and temperature $T_{x}$, which are both modulated at the detuning frequency $\Omega$ by the beating of the pump and probe waves. The lattice temperature $T_{L}$ is restored in each subband by electron-phonon scattering with a characteristic lifetime $\tau_{h x}\left(>\tau_{1}\right)$; this time constant is associated with carrier heating and is described by the second diagonal term, where $f_{x}^{L}=f_{x}\left(N, T_{L}\right)$.

The off-diagonal term between $|a\rangle$ and $|b\rangle$ accounts for the loss of phase coherence of the tunneling oscillations. This is due to carrier-carrier scattering and other (slower) mechanisms such as carrier-phonon and interface roughness, so that in general the dephasing time constant $\tau_{d}$ is expected to be shorter than $\tau_{1}$. Finally, the off-diagonal elements coupling $|v\rangle$ to either $|a\rangle$ or $|b\rangle$ describe the damping of the interband optical dipole oscillations. ${ }^{1} R_{\text {coll }}$ may be readily converted to the $\{|r\rangle,|l\rangle,|v\rangle\}$ representation using (1) and the definition of the density matrix.

Since $1 / \tau_{s}$ and $1 / \tau_{h x}$ are typically much smaller than $1 / \tau_{1 x}, 1 / \tau_{2}, 1 / \tau_{d}$, the terms accounting for spontaneous recombination and relaxation to the lattice temperature can be

${ }^{1}$ In [11], a factor of $1 / \sqrt{2}$ was erroneously introduced in the damping terms for $\rho_{a v}$ and $\rho_{b v}$. However, the expression for $R_{\text {coll }}$ in the $\{|r\rangle,|l\rangle,|v\rangle\}$ representation used in the analysis presented there was correct, so that the error did not affect any of the subsequent results. omitted from the density matrix dynamic equations. Carrier density pulsations and carrier heating are included in this formulation through the dependence of $f_{x}$ on $N_{x}$ and $T_{x}$, which may be approximated as follows:

$$
\begin{aligned}
f_{x}(t)= & \bar{f}_{x}+\frac{\partial f_{x}}{\partial N_{x}}\left(N_{x}^{(\Omega)} e^{-i \Omega t}+\text { c.c. }\right) \\
& +\frac{\partial f_{x}}{\partial T_{x}}\left(T_{x}^{(\Omega)} e^{-i \Omega t}+\text { c.c. }\right)
\end{aligned}
$$

where the overbar denotes the quasiequilibrium Fermi distributions determined by the pumping level. $N_{x}^{(\Omega)}$ and $T_{x}^{(\Omega)}$ are the carrier density and temperature oscillation amplitudes and can be evaluated from the respective rate equations, which can be immediately derived from (6), (7), and (10) below. This procedure is described in [17], and will not be considered here, since, as will be discussed in Section V, spectral hole burning is expected to be the dominant FWM process under the conditions of interest here.

Finally, we notice that the term proportional to $\mu_{r}$ in the Hamiltonian of (2) only introduces nonresonant contributions (at optical frequencies) to the density matrix time evolution. Neglecting these terms, we then arrive at the following set of coupled equations:

$$
\begin{aligned}
\frac{d \rho_{v}}{d t}= & -i \frac{\mu_{l v}}{\hbar} \mathcal{E}(t)\left(\rho_{v l}-\rho_{l v}\right)-\frac{\rho_{v}-f_{v}}{\tau_{1}} \\
\frac{d \rho_{l}}{d t}= & -i \frac{\mu_{l v}}{\hbar} \mathcal{E}(t)\left(\rho_{l v}-\rho_{v l}\right)-i \frac{\omega_{t}}{2}\left(\rho_{l r}-\rho_{r l}\right) \\
& -\frac{\rho_{l}+\rho_{r}-\left(f_{b}+f_{a}\right)}{2 \tau_{1}}-\frac{\rho_{l}-\rho_{r}}{2 \tau_{d}} \\
\frac{d \rho_{l v}}{d t}= & -i \frac{\mu_{l v}}{\hbar} \mathcal{E}(t)\left(\rho_{l}-\rho_{v}\right)-i \omega_{0} \rho_{l v} \\
& +i \frac{\omega_{t}}{2} \rho_{r v}-\frac{\rho_{l v}}{\tau_{2}} \\
\frac{d \rho_{r v}}{d t}= & -i \frac{\mu_{l v}}{\hbar} \mathcal{E}(t) \rho_{r l}-i \omega_{0} \rho_{r v} \\
& +i \frac{\omega_{t}}{2} \rho_{l v}-\frac{\rho_{r v}}{\tau_{2}}, \\
\frac{d \rho_{r}}{d t}= & -i \frac{\omega_{t}}{2}\left(\rho_{r l}-\rho_{l r}\right)-\frac{\rho_{r}+\rho_{l}-\left(f_{b}+f_{a}\right)}{2 \tau_{1}} \\
& -\frac{\rho_{r}-\rho_{l}}{2 \tau_{d}}, \\
\frac{d \rho_{r l}}{d t}= & -i \frac{\mu_{l v}}{\hbar} \mathcal{E}(t) \rho_{r v}-i \frac{\omega_{t}}{2}\left(\rho_{r}-\rho_{l}\right) \\
& -\frac{\rho_{r l}+\rho_{l r}-\left(f_{b}-f_{a}\right)}{2 \tau_{1}}-\frac{\rho_{r l}-\rho_{l r}}{2 \tau_{d}}
\end{aligned}
$$

where $\omega_{0}$ is the interband transition frequency in the left well in the absence of coupling, and, for simplicity, we took the carrier-carrier scattering time constant $\tau_{1}$ to be the same in all subbands.

$$
R_{\mathrm{coll}}=-\left[\begin{array}{ccc}
\frac{1}{\tau_{1 a}}\left(\rho_{a}-f_{a}\right)+\frac{1}{\tau_{h a}}\left(\rho_{a}-f_{a}^{L}\right) & \frac{1}{\tau_{d}} \rho_{a b} & \frac{\rho_{a v}}{\tau_{2}} \\
\frac{1}{\tau_{d}} \rho_{b a} & \frac{1}{\tau_{1 b}}\left(\rho_{b}-f_{b}\right)+\frac{1}{\tau_{h b}}\left(\rho_{b}-f_{b}^{L}\right) & \frac{\rho_{b v}}{\tau_{2}} \\
\frac{\rho_{v a}}{\tau_{2}} & \frac{\rho_{v b}}{\tau_{2}}\left(\rho_{v}-f_{v}\right)+\frac{1}{\tau_{h v}}\left(\rho_{v}-f_{v}^{L}\right)
\end{array}\right]
$$




\section{SOlUTION OF THE DENSTTY \\ MATRIX DYNAMIC EQUATIONS}

The optical field (3) induces in the active region of the TWA a macroscopic polarization

$$
\begin{aligned}
\mathcal{P}(t)= & P_{p} \exp \left(-i \omega_{p} t\right)+P_{q} \exp \left(-i \omega_{q} t\right)+P_{s} \exp \left(-i \omega_{s} t\right) \\
& +P^{(\Omega)} \exp (-i \Omega t)+\text { c.c. }
\end{aligned}
$$

which can be expressed as

$$
\mathcal{P}(t)=\frac{1}{V} \sum_{k} \mu_{l v}\left(\rho_{l v}+\rho_{v l}\right)+\mu_{r} \rho_{r},
$$

where $V$ is the volume of the active region, and the summation may be replaced by an integral over the appropriate density of states. All the harmonic components of the polarization density can then be immediately derived from the solutions to (6)-(11) for $\rho_{i v}$ and $\rho_{r}$.

Consistent with the discussion of the previous section, we look for solutions of the form

$$
\begin{aligned}
\rho_{x v}(t)= & \rho_{x v}^{\left(\omega_{p}\right)} \exp \left(-i \omega_{p} t\right)+\rho_{x v}^{\left(\omega_{q}\right)} \exp \left(-i \omega_{q} t\right) \\
& +\rho_{x v}^{\left(\omega_{s}\right)} \exp \left(-i \omega_{s} t\right), \quad(x=r, l) \\
\rho_{x}(t)= & \rho_{x}^{(0)}+\left[\rho_{x}^{(\Omega)} \exp (-i \Omega t)+c . c .\right], \quad(x=r, l, v) \\
\rho_{r l}= & \rho_{r l}^{(0)}+\rho_{r l}^{(\Omega)} \exp (-i \Omega t)+\dot{\rho}_{r l}^{(-\Omega)} \exp (+i \Omega t) .
\end{aligned}
$$

Substituting these expressions in (8) and (9) gives

$$
\begin{aligned}
\rho_{l v}^{\left(\omega_{p}\right)} \cong & -i \frac{\mu_{l v}}{\hbar} \tilde{\chi}^{\prime}\left(\omega_{p}\right)\left\{\left(\rho_{l}^{(0)}-\rho_{v}^{(0)}\right) E_{p}+i \frac{\omega_{t} \tilde{\chi}\left(\omega_{p}\right)}{2} \rho_{r l}^{(0)} E_{p}\right\}, \\
\rho_{l v}^{\left(\omega_{q}\right)}= & -i \frac{\mu_{l v}}{\hbar} \tilde{\chi}^{\prime}\left(\omega_{q}\right) \\
\times & \times\left\{\left(\rho_{l}^{(0)}-\rho_{v}^{(0)}\right) E_{q}+\left(\rho_{l}^{(\Omega)}-\rho_{v}^{(\Omega)}\right)^{*} E_{p}\right. \\
& \left.+i \frac{\omega_{t} \tilde{\chi}\left(\omega_{q}\right)}{2}\left[\rho_{r l}^{(0)} E_{q}+\rho_{r l}^{(-\Omega)} E_{p}\right]\right\}, \\
\rho_{l v}^{\left(\omega_{s}\right)}= & -i \frac{\mu_{l v}}{\hbar} \tilde{\chi}^{\prime}\left(\omega_{s}\right) \\
& \times\left\{\left(\rho_{l}^{(0)}-\rho_{v}^{(0)}\right) E_{s}+\left(\rho_{l}^{(\Omega)}-\rho_{v}^{(\Omega)}\right) E_{p}\right. \\
& \left.+i \frac{\omega_{t} \tilde{\chi}\left(\omega_{s}\right)}{2}\left[\rho_{r l}^{(0)} E_{s}+\rho_{r l}^{(\Omega)} E_{P}\right]\right\},
\end{aligned}
$$

where we have defined

$$
\tilde{\chi}(\omega)=\frac{\tau_{2}}{1-i \tau_{2}\left(\omega-\omega_{0}\right)}, \quad \tilde{\chi}^{\prime}(\omega)=\frac{\tilde{\chi}(\omega)}{1+\left(\frac{\omega_{t} \tilde{\chi}(\omega)}{2}\right)^{2}},
$$

and, in writing (17), we have used the fact that the pump wave is much stronger than any other signal. Similar expressions are found for the harmonic components of $\rho_{r v}$.

These equations can then be used to eliminate $\rho_{l v}$ and $\rho_{r v}$ from (6), (7), and (11) so that the problem reduces to the determination of the diagonal elements and $\rho_{r l}$. Neglecting from (17)-(19) (and from the corresponding expressions for $\rho_{r v}$ ) the terms proportional to $\rho_{x}^{(\Omega)}$ and replacing $\rho_{x}^{(0)}$ with the corresponding quasiequilibrium Fermi distributions, which amounts to keeping the solution to first order in $\left|E_{p}\right|^{2},(6)$,
(7), and (11) become

$$
\begin{aligned}
\frac{d \rho_{v}}{d t}= & {\left[A+B e^{-i \Omega t}+\text { c.c. }\right]-\frac{\rho_{v}-f_{v}}{\tau_{1}} } \\
\frac{d \rho_{l}}{d t}= & {\left[A+B e^{-i \Omega t}+\text { c.c. }\right]-i \frac{\omega_{t}}{2}\left(\rho_{l r}-\rho_{r l}\right) } \\
& -\frac{\rho_{l}+\rho_{r}-\left(f_{b}+f_{a}\right)}{2 \tau_{1}}-\frac{\rho_{l}-\rho_{r}}{2 \tau_{d}} \\
\frac{d \rho_{\boldsymbol{r} l}}{d t}= & {\left[A^{\prime}+B^{\prime} e^{-i \Omega t}+B^{\prime \prime} e^{+i \Omega t}\right]-i \frac{\omega_{t}}{2}\left(\rho_{r}-\rho_{l}\right) } \\
& -\frac{\rho_{r l}+\rho_{l r}-\left(f_{b}+f_{a}\right)}{2 \tau_{1}}-\frac{\rho_{r l}-\rho_{l r}}{2 \tau_{d}} .
\end{aligned}
$$

Here, the parameters $A, A^{\prime}, B, B^{\prime}$, and $B^{\prime \prime}$ denote the strength of the spectral hole burning process, and are given by

$$
\begin{aligned}
& A=-\frac{\mu_{l v}^{2}}{\hbar^{2}} \Delta f \tilde{\chi}\left(\omega_{p}\right)\left|E_{p}\right|^{2}, \quad A^{\prime}=i \frac{\omega_{t} \tilde{\chi}^{\prime}\left(\omega_{p}\right)}{2} A \\
& B=-\frac{\mu_{l v}^{2}}{\hbar^{2}} \Delta f\left[\left(\tilde{\chi}^{\prime}\left(\omega_{p}\right)+\tilde{\chi}^{\prime *}\left(\omega_{q}\right)\right) E_{p} E_{q}^{*}\right. \\
& \left.+\left(\tilde{\chi}^{\prime}\left(\omega_{s}\right)+\tilde{\chi}^{\prime *}\left(\omega_{p}\right)\right) E_{p}^{*} E_{s}\right] \\
& B^{\prime}=-i \frac{\omega_{t}}{2} \frac{\mu_{l v}^{2}}{\hbar^{2}} \Delta f\left[\tilde{\chi}^{\prime}\left(\omega_{p}\right) \tilde{\chi}\left(\omega_{p}\right) E_{p} E_{q}^{*}\right. \\
& \left.+\tilde{\chi}^{\prime}\left(\omega_{s}\right) \tilde{\chi}\left(\omega_{s}\right) E_{p}^{*} E_{s}\right] \\
& B^{\prime \prime}=-i \frac{\omega_{t}}{2} \frac{\mu_{l v}^{2}}{\hbar^{2}} \Delta f\left[\tilde{\chi}^{\prime}\left(\omega_{p}\right) \tilde{\chi}\left(\omega_{p}\right) E_{p} E_{s}^{*}\right. \\
& \left.+\tilde{\chi}^{\prime}\left(\omega_{q}\right) \tilde{\chi}\left(\omega_{q}\right) E_{p}^{*} E_{q}\right]
\end{aligned}
$$

where $\Delta f$ is the Fermi inversion factor, $\Delta f=\left(f_{b}+f_{a}\right) / 2-f_{v}$. The occupation probability of the valence band states $\rho_{v}$ can then be readily found by using (15) and (5) in (21); the result is

$$
\begin{aligned}
& \rho_{v}^{(0)}=\vec{f}_{v}-\frac{\tau_{1}}{2}(A+\text { c.c. }), \\
& \rho_{v}^{(\Omega)}=-\frac{1}{1-i \tau_{1} \Omega}\left(\tau_{l} B-\frac{\partial f_{v}}{\partial N_{v}} N_{v}^{(\Omega)}-\frac{\partial f_{v}}{\partial T_{v}} T_{v}^{(\Omega)}\right) .
\end{aligned}
$$

In order to obtain the corresponding expressions for $\rho_{l}, \rho_{r}$, and $r$, it is necessary to simultaneously solve (10), (22), and (23). To this end, it is convenient to introduce a vector representation by defining the new variables [12]

$$
\begin{aligned}
& \rho_{0}=\rho_{l}+\rho_{r}, \quad \vec{\rho}=\hat{\mathbf{x}} \rho_{x}+\hat{\mathbf{y}} \rho_{y}+\hat{\mathbf{z}} \rho_{z}, \\
& \rho_{x}=\rho_{l}-\rho_{r}, \quad \rho_{y}=i\left(\rho_{r l}-\rho_{l r}\right), \quad \rho_{z}=\rho_{l r}+\rho_{r l}
\end{aligned}
$$

and transform the resulting equations to a reference frame rotating about the " $z$ axis" with radian frequency $\omega_{t}$. The result gives the following steady-state solutions:

$$
\begin{aligned}
\rho_{l}^{(0)}= & \frac{\bar{f}_{b}+\bar{f}_{a}}{2}+\frac{\tau_{1}}{2}(A+\text { c.c. }) \\
& +\frac{\tau_{d}}{4}\left(\frac{A+A^{\prime}}{1-i \omega_{t} \tau_{d}}+\frac{A-A^{\prime}}{1+i \omega_{t} \tau_{d}}+\text { c.c. }\right) \\
\rho_{l}^{(\Omega)}= & \frac{\tau_{1}}{2} \frac{B+C}{1-i \Omega \tau_{1}} \\
& +\frac{\tau_{d}}{4}\left[\frac{B+B^{\prime}-B^{\prime \prime *}}{1-i\left(\Omega+\omega_{t}\right) \tau_{d}}+\frac{B-B^{\prime}+B^{\prime \prime *}}{1-i\left(\Omega-\omega_{t}\right) \tau_{d}}\right] \\
\rho_{r l}^{(0)}= & \frac{\bar{f}_{b}-\bar{f}_{a}}{2}+\frac{\tau_{1}}{2}\left(A^{\prime}+\text { c.c. }\right) \\
& +\frac{\tau_{d}}{4}\left(\frac{A+A^{\prime}}{1-i \omega_{t} \tau_{d}}+\frac{A-A^{\prime}}{1+i \omega_{t} \tau_{d}}-\text { c.c. }\right),
\end{aligned}
$$




$$
\begin{aligned}
\rho_{r l}^{(\Omega)}= & \frac{\tau_{1}}{2} \frac{B^{\prime}+B^{\prime \prime *}}{1-i \Omega \tau_{1}} \\
& +\frac{\tau_{d}}{4}\left[\frac{B+B^{\prime}-B^{\prime \prime *}}{1-i\left(\Omega+\omega_{t}\right) \tau_{d}}-\frac{B-B^{\prime}+B^{\prime \prime *}}{1-i\left(\Omega-\omega_{t}\right) \tau_{d}}\right] .
\end{aligned}
$$

The corresponding expressions for $\rho_{r}$ are the same as those for $\rho_{l}$, except that in both cases the sign of all terms proportional to $\tau_{d}$ is reversed. The parameter $C$ defined in (32) is given by

$$
C=\frac{1}{\tau_{1}}\left(\frac{\partial f_{a}}{\partial N_{a}} N_{a}^{(\Omega)}+\frac{\partial f_{a}}{\partial T_{a}} T_{a}^{(\Omega)}+\frac{\partial f_{b}}{\partial N_{b}} N_{b}^{(\Omega)}+\frac{\partial f_{b}}{\partial T_{b}} T_{b}^{(\Omega)}\right),
$$

and accounts for carrier density pulsations and carrier heating in the conduction subbands.

\section{Four-Wave MiXING CONVERSION EFFICIENCY}

In order to estimate the FWM conversion efficiency of the ASCQW structure, we need to evaluate the harmonic component of the polarization density at $\omega_{s}$, which provides the source of the converted signal. Comparison of (12), (13), (14), and (19) shows that this can be cast in the form

$$
\begin{aligned}
P_{s}= & -\frac{i}{V} \sum_{k} \frac{\mu_{l v}^{2}}{\hbar} \tilde{\chi}^{\prime}\left(\omega_{s}\right) \\
& \times\left\{\left(\rho_{l}^{(0)}-\rho_{v}^{(0)}\right) E_{s}+\left(\rho_{l}^{(\Omega)}-\rho_{v}^{(\Omega)}\right) E_{p}\right. \\
& \left.+i \frac{\omega_{t} \tilde{\chi}\left(\omega_{s}\right)}{2}\left[\rho_{r l}^{(0)} E_{s}+\rho_{r l}^{(\Omega)} E_{p}\right]\right\}
\end{aligned}
$$

For the optical interaction under consideration, $P_{s}$ has the general form [17] $P_{s}=\varepsilon_{0} \chi_{L} E_{s}+\varepsilon_{0} \chi_{\mathrm{FWM}} E_{p}^{2} E_{q}^{*}$, where the second term is responsible for FWM, and in general has contributions from carrier density pulsations, carrier heating, and spectral hole burning, $\chi_{\mathrm{FWM}}=\chi_{\mathrm{CDP}}+\chi_{\mathrm{CH}}+\chi_{\mathrm{SHB}}$. The different terms in $\chi_{\mathrm{FWM}}$ can be immediately evaluated by substitution of (28)-(29) and (31)-(34) in (36).

At detuning frequencies on the order of $\omega_{t}$, spectral hole burning is expected to be the dominant FWM process, so that the magnitude squared of $\chi_{\mathrm{SHB}}$ is a good estimate of the overall FWM power conversion efficiency in this detuning range. This approximation is expected to be particularly valid at low temperatures for the following reason: the contributions to $\chi_{\mathrm{FWM}}$ from carrier density pulsations and carrier heating are proportional to the rate of change of the quasiequilibrium distributions with carrier number and temperature respectively, which, as the temperature is lowered, become smaller everywhere in $k$ space (away from the Fermi surface). As pointed out previously, the spectral hole burning contribution to the modulation of the occupation probabilities $\rho_{l}$ and $\rho_{v}$ is accounted for by the terms proportional to $A, A^{\prime}, B, B^{\prime}$, and $B^{\prime \prime}$ in (28)-(29) and (31)-(34); retaining only these terms upon substitution in (36), we obtain

$$
\begin{aligned}
\chi_{\mathrm{SHB}}= & \frac{i}{\varepsilon_{0}} \\
\times & \frac{1}{V} \sum_{k} \frac{\mu_{l v}^{4}}{\hbar^{3}} \Delta f \tilde{\chi}^{\prime}\left(\omega_{s}\right) \\
& \frac{\tau_{1}}{1-i \Omega \tau_{1}} L_{1}\left(\Omega ; \omega_{t}\right)+\frac{1}{4} \frac{\tau_{d}}{1-i\left(\Omega+\omega_{t}\right) \tau_{d}} \\
& \times\left(1+i \frac{\omega_{t} \tilde{\chi}\left(\omega_{s}\right)}{2}\right) L_{2}\left(\Omega ; \omega_{t}\right)
\end{aligned}
$$

$$
\begin{aligned}
& +\frac{1}{4} \frac{\tau_{d}}{1-i\left(\Omega-\omega_{t}\right) \tau_{d}} \\
& \left.\times\left(1-i \frac{\omega_{t} \tilde{\chi}\left(\omega_{s}\right)}{2}\right) L_{2}\left(\Omega ;-\omega_{t}\right)\right\}
\end{aligned}
$$

where

$$
\begin{aligned}
L_{1}\left(\Omega ; \omega_{t}\right)= & \tilde{\chi}^{\prime}\left(\omega_{p}\right)+\tilde{\chi}^{\prime *}\left(\omega_{q}\right)-\frac{\omega_{t}^{2}}{4} \tilde{\chi}\left(\omega_{s}\right) \\
& \times\left(\tilde{\chi}^{\prime}\left(\omega_{p}\right) \tilde{\chi}\left(\omega_{p}\right)-\tilde{\chi}^{\prime *}\left(\omega_{q}\right) \tilde{\chi}^{*}\left(\omega_{q}\right)\right) \\
L_{2}\left(\Omega ; \omega_{t}\right)= & \tilde{\chi}^{\prime}\left(\omega_{p}\right)+\tilde{\chi}^{* *}\left(\omega_{q}\right)+i \frac{\omega_{t}}{2} \\
& \times\left(\tilde{\chi}^{\prime}\left(\omega_{p}\right) \tilde{\chi}\left(\omega_{p}\right)+\tilde{\chi}^{\prime *}\left(\omega_{q}\right) \tilde{\chi}^{*}\left(\omega_{q}\right)\right)
\end{aligned}
$$

The expression for $\chi_{\mathrm{SHB}}$ in (37) reduces to that of a bulk or uncoupled-well amplifier [17] as $\omega_{t}$ approaches zero (and $\tau_{d}=\tau_{1}$ ). In the presence of interwell coupling, however, two effects contribute to significantly enhance the magnitude of $\chi_{\text {SHB }}$ for detuning frequencies near $\omega_{t}$. First, consistent with the interpretation of the spectral hole producing the gratings as a harmonic oscillator driven at its natural frequency, a resonance is introduced at $\omega_{t}$ for both negative and positive detuning rates, as indicated by the complex Lorentzian in the second and third terms in curly brackets, respectively. Furthermore, the overall lineshape of the third-order transition leading to generation of the converted-signal photons is also modified by the interwell coupling in a way so as to enhance such transition. Physically, this is due to the presence of an additional contribution to the dipole between $|l\rangle$ and $|v\rangle$ (embodied in the last term of (17)-(19)), arising from the time-harmonic variation of the population of the upper level $|l\rangle$ due to the tunneling oscillations. (Note: the latter effect was neglected in [11], where $\tau_{2}$ was assumed to be smaller than any other time constant. In particular, for $\tau_{2} \omega_{t} \ll 1$, the dipole transitions are damped out in a time interval much smaller than the tunneling oscillation period, so that this additional contribution to $\rho_{l v}$ becomes negligible. In this limit, (37) reduces to the simpler expression for $\chi_{\mathrm{SHB}}$ given there. All the basic results are indeed contained in that expression; however, the full solution reported here shows that such contribution considerably enhances the overall effect).

For a numerical estimate, we considered an ASCQW optical amplifier based on the InGaAsP material system and consisting of pairs of $40-\AA$ compressive wells and $160-\AA$ tensile wells separated by a $20-\AA$ barrier, with carrier density $N=2.3$ $\times 10^{24} \mathrm{~m}^{-3}$. We assumed alloy compositions appropriate to operation at $1.55 \mu \mathrm{m}$. The interband dipole moment of the tensile strained well (the one providing gain in this context) was then estimated to be $\mu_{i v}=\mathrm{e} \times 11.5 \AA$, giving a peak gain coefficient of approximately $5800 \mathrm{~cm}^{-1}$ at room temperature. The energy separation between the valence subband edges in the two wells was found to be (under zero bias condition) $20 \mathrm{meV}$ at $k=0$ (and larger at finite $k$ due to the different dispersion in the two oppositely strained wells), which amply justifies neglect of hole interwell coupling. Finally, we assumed $\omega_{t}=4 \mathrm{THz}$.

The magnitude squared of $\chi_{\mathrm{SHB}}(\Omega)$ at terahertz detuning rates is plotted in Fig. 2 , where $\chi_{\mathrm{SHB}}$ is evaluated in MKS units and plotted in decibels for convenience. The relaxation 


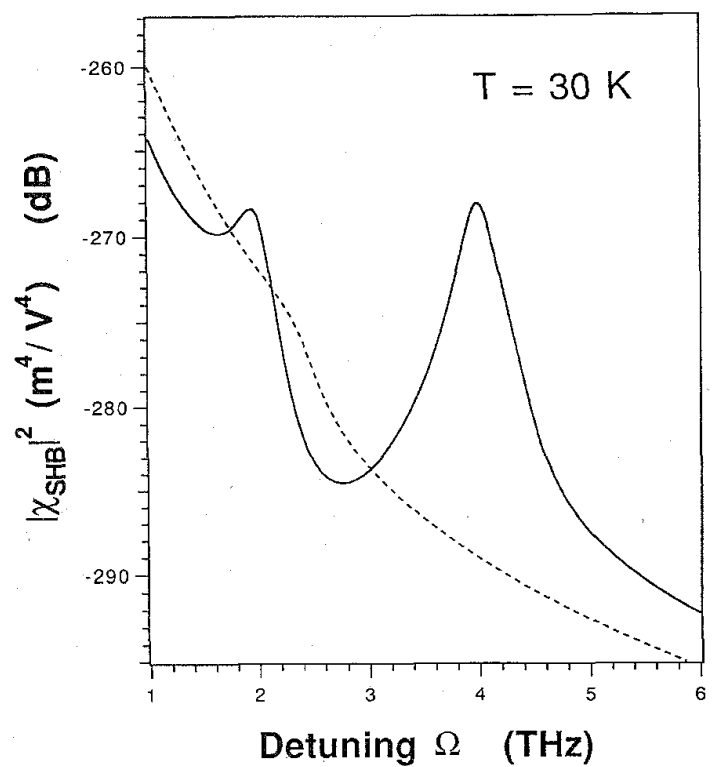

Fig. 2. Magnitude squared of the spectral-hole burning contribution to the FWM susceptibility in the ASCQW structure (solid line) and in an uncoupled-well amplifier (dashed line) versus detuning frequency, for $\tau_{1}=\tau_{2}=\tau_{d}=1 \mathrm{ps}, \omega_{t}=4 \mathrm{THz}$. In this detuning range, $\left|\chi_{\text {SHB }}\right|^{2}$ gives a good estimate of the overall power conversion efficiency.

time constants $\tau_{1}, \tau_{2}$, and $\tau_{d}$ were taken equal to $1 \mathrm{ps}$, as appropriate to a temperature of around $30^{\circ} \mathrm{K}$ and operation near the Fermi edge [18]. For comparison, $\left|\chi_{\mathrm{SHB}}\right|^{2}$ in the limit of no coupling between the wells is also shown (dotted line). A pronounced peak, preceded by a dip, is clearly introduced at the resonance frequency, giving rise to a maximum increase in the conversion efficiency of approximately two orders of magnitude at $\Omega=\omega_{t}$. The asymmetry between the peak and the dip is due to the $\Omega$ dependence of the lineshape function of (39). Note that at detuning frequencies below $\omega_{t},\left|\chi_{\mathrm{SHB}}\right|^{2}$ is smaller in the ASCQW structure than in the uncoupled case by approximately $4 \mathrm{~dB}$; this is attributed to the reduction of the (time-average) amplitude of the "spectral hole" by the oscillations across the barrier. However, the resulting decrease in the overall FWM efficiency becomes negligible at subterahertz detuning rates, where the relative contribution of carrier heating and carrier density pulsations is enhanced over that of spectral hole burning.

The size of the resonance peak is obviously limited by the damping rate $1 / \tau_{d}$. This is illustrated in Fig. 3, where the ratio of $\left|\chi_{\mathrm{SHB}}\right|^{2}$ to its value in the uncoupled case $\left(\left|\chi_{\mathrm{SHB}}^{\mathrm{UNC}}\right|^{2}\right)$ is plotted for different values of $\tau_{d}$. As shown in this figure, the effect of the interwell coupling should be detectable for $\tau_{d}$ as small as a few hundred femtoseconds, indicating that higher temperature operation may be possible.

\section{GENERATION OF FAR-INFRARED RADIATION}

The terahertz spectral region $(1-10 \mathrm{THz}$ ) still awaits the development of convenient sources of coherent narrowband radiation, which may be quite useful for applications such as local oscillator sources in high resolution laser heterodyne spectrometers. In the past few years, several schemes have

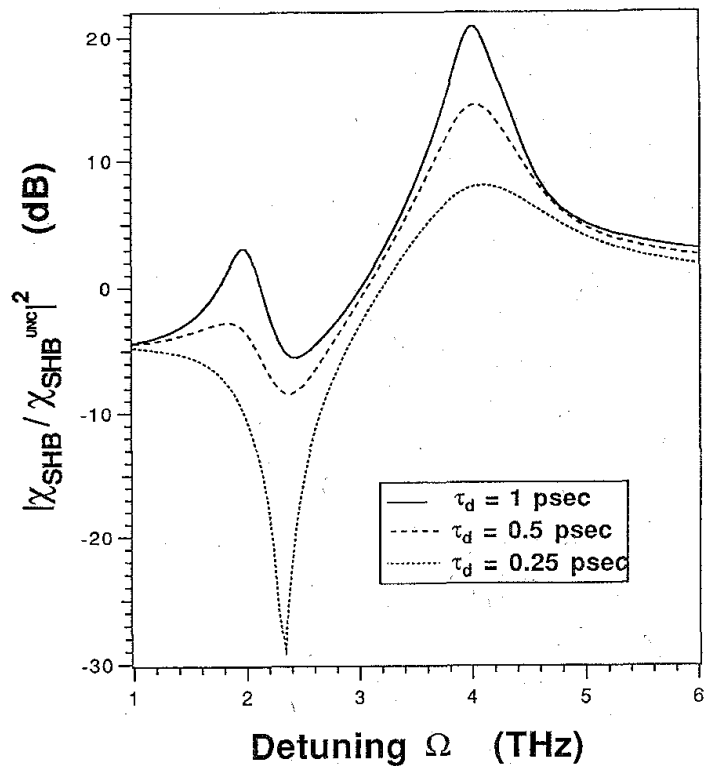

Fig. 3. Magnitude squared of the spectral-hole burning contribution to the FWM susceptibility in the ASCQW structure normalized to its value in the uncoupled-well limit versus detuning frequency, for $\omega_{t}=4 \mathrm{THz}$, and $\tau_{1}=\tau_{2}=\tau_{d}=1,0.5,0.25 \mathrm{ps}$, corresponding to an estimated temperature of 30,60 , and $90 \mathrm{~K}$, respectively [18].

been proposed [19]-[21], followed by promising experimental demonstrations [22]-[24]. In this section, we consider the generation of terahertz radiation by the steady-state electronic wave packet oscillations across the conduction-band barrier. The results suggest a novel scheme for generation of far infrared radiation, based on wave mixing in the ASCQW structure.

The harmonic component of the polarization density at $\Omega$ is given by

$$
P^{(\Omega)}=\frac{1}{V} \sum_{k} \mu_{r} \rho_{r}^{(\Omega)}
$$

and can be immediately evaluated using the expression for $\rho_{r}^{(\Omega)}$ corresponding to (32). For simplicity, we will again include only the contribution from spectral hole burning (the terms of (32) proportional to the parameters $B, B^{\prime}, B^{\prime \prime}$ ), and use the fact that the conjugate signal is much weaker than the pump and probe waves so as to enable neglect of the term proportional to $E_{p}^{*} E_{s}$ from the expression for $B, B^{\prime}, B^{\prime \prime}$. Then, defining the appropriate (second-order) susceptibility $\chi_{\mathrm{TH}}$ so that $P^{(\Omega)}=\varepsilon_{0} \chi_{\mathrm{TH}} E_{p} E_{q}^{*}$, we find

$$
\begin{aligned}
\chi_{\mathrm{TH}}= & -\frac{1}{\varepsilon_{0}} \frac{1}{V} \sum_{k} \frac{\mu_{r} \mu_{l v}^{2}}{\hbar^{2}} \Delta f \\
& \times\left\{\frac{1}{2} \frac{\tau_{1}}{1-i \Omega \tau_{1}}\left[\tilde{\chi}^{\prime}\left(\omega_{p}\right)+\tilde{\chi}^{\prime *}\left(\omega_{q}\right)\right]\right. \\
& -\frac{1}{4} \frac{\tau_{d}}{1-i\left(\Omega+\omega_{t}\right) \tau_{d}} L_{2}\left(\Omega ; \omega_{t}\right) \\
& \left.-\frac{1}{4} \frac{\tau_{d}}{1-i\left(\Omega-\omega_{t}\right)} L_{2}\left(\Omega ;-\omega_{t}\right)\right\}
\end{aligned}
$$




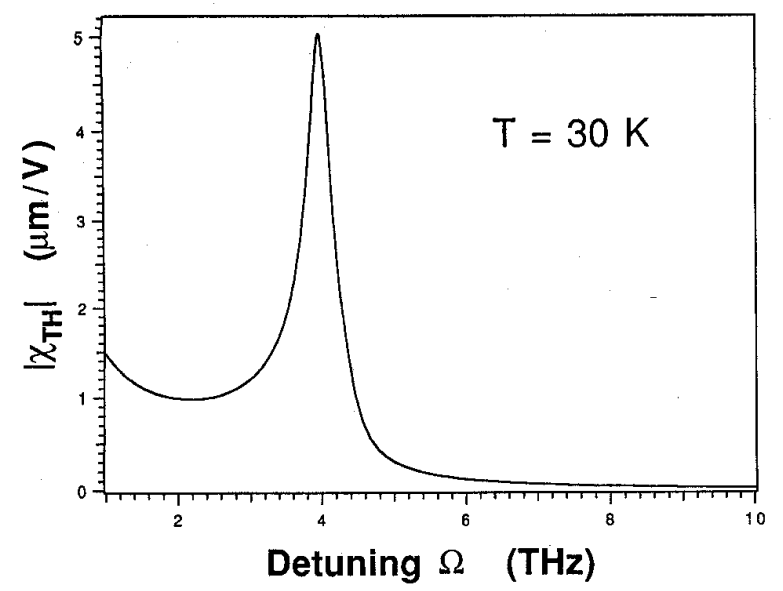

Fig. 4. Magnitude of the second-order susceptibility associated with generation of terahertz radiation versus detuning frequency for $\tau_{1}=\tau_{2}=\tau_{d}=1 \mathrm{ps}$ $(T=30 \mathrm{~K})$. An increase of approximately one order of magnitude is observed at the resonance frequency $\omega_{t}=4 \mathrm{THz}$, due to the interwell coupling.

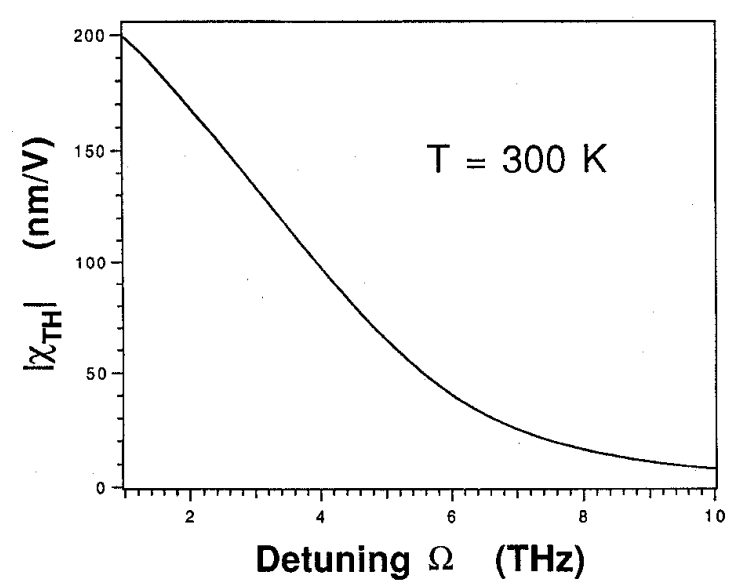

Fig. 5. Magnitude of the second-order susceptibility associated with generation of terahertz radiation versus detuning frequency for room temperature operation, for which $\tau_{1}=\tau_{2}=\tau_{d}=50 \mathrm{fs}$.

where $L_{2}$ is defined in (39). The magnitude of $\chi_{\mathrm{TH}}$ is plotted in Fig. 4 versus detuning frequency, for $\mu_{r}=\mathrm{e} \times 110 \AA$, and assuming $T=30 \mathrm{~K}$ as in Section. V. Again consistent with the harmonic oscillator picture, there appears a peak about the resonance frequency $\omega_{t}(4 \mathrm{THz})$, where $\left|\chi_{\mathrm{TH}}\right|=5 \cdot 10^{-6} \mathrm{~m} / \mathrm{V}$, with lineshape determined by the dephasing time constant $\tau_{d}$. In Fig. 5, $\left|\chi_{\mathrm{TH}}(\Omega)\right|$ is plotted under room temperature conditions, for which we took $\tau_{1}=\tau_{d}=\tau_{2}=50 \mathrm{fs}$. The peak at resonance is now completely smeared out due to the ultrafast dephasing of the tunneling oscillations. However, the continuous excitation of such oscillations enabled by our proposed scheme still provides a large $\left|\chi_{\mathrm{TH}}(\Omega)\right|$, on the order of $10^{-7} \mathrm{~m} / \mathrm{V}$ for $\Omega$ up to about $4 \mathrm{THz}$.

In conclusion, we point out that, while both the wavelengthconverted signal and the terahertz signal are simultaneously generated in the ASCQW structure by the nonlinear interactions considered here, optimization of one effect (through design considerations involving the waveguide structure, operating wavelength, pumping level, etc.) may be accompanied by a reduction of the other. In particular, for efficient generation of far infrared radiation at the output of the device, one has to deal with phase-matching considerations and the strong absorption at terahertz frequencies due to freecarrier absorption (phase-matching is much less of a problem in the case of four-wave mixing, see e.g., [7]). The phase mismatch between the input waves and the generated terahertz signal may be minimized by taking advantage of the acousticphonon contribution to the dielectric response at terahertz frequencies. Because of the variation of such contribution with both frequency and wavevector, one may envision a generation scheme in which pump, probe, and terahertz wave propagate along different directions (parallel to the heterojunction plane) for which the phase-matching conditon is satisfied. Similarly, as suggested by recent work [19], [21], [24], free-carrier absorption may be minimized by appropriate design of the overall device structure. The scheme for generation of far infrared radiation described above seems then very promising in view of the large nonlinearity involved. In fact, in the work of [24], the authors report extremely efficient generation of $2.5 \mathrm{THz}$ radiation from difference frequency mixing in (Al)GaAs multiple quantum-well waveguides associated with an estimated $\chi^{(2)}$ comparable to the value for $\chi_{\text {FIR }}$ computed here. It would then be quite interesting to consider the design and practical implementation of a parametric oscillator based on NDFWM in the ASCQW structure.

\section{REFERENCES}

[1] L. F. Tiemeijer, "Effects of nonlinear gain on four-wave mixing and asymmetric gain saturation in a semiconductor laser amplifier," Appl. Phys. Lett., vol. 59, pp. 499-501, 1991.

[2] K. Kikuchi, M. Kakui, C. E. Zah, and T. P. Lee, "Observation of highly nondegenerate four-wave mixing in $1.5 \mu \mathrm{m}$ traveling-wave semiconductor optical amplifiers and estimation of nonlinear gain coefficient," IEEE J. Quantum Electron., vol. 28, pp. 151-156, 1992.

[3] J. Zhou, N. Park, J. W. Dawson, K. J. Vahala, M. A. Newkirk, and B. I. Miller, "Terahertz four-wave mixing spectroscopy for study of ultrafast dynamics in a semiconductor optical amplifier," Appl. Phys. Lett., vol. 63, pp. $1179-1181,1993$.

[4] A. D'Ottavi, E. Iannone, A. Mecozzi, S. Scotti, P. Spano, J. Landreau, A. Ougazzaden, and J. C. Bouley, "Investigation of carrier heating and spectral hole burning in semiconductor amplifiers by highly nondegenerate four-wave mixing," Appl. Phys. Lett., vol. 64, pp. 2492-2494, 1994.

[5] A. Uskov, J. Mørk, J. Mark, M. C. Tatham, and G. Sherlock, "Terahertz four-wave mixing in semiconductor optical amplifiers: Experiment and theory," Appl. Phys. Lett., vol. 65, pp. 944-946, 1994.

[6] M. C. Tatham, G. Sherlock, and L. D. Westbrook, "20-nm optical wavelength conversion using nondegenerate four-wave mixing," IEEE Photon. Technol. Lett., vol. 5, pp. 1303-1306, 1993.

[7] J. Zhou, N. Park, J. W. Dawson, K. J. Vahala, M. A. Newkirk, and B. I. Miller, "Efficiency of broadband four-wave mixing wavelength conversion using semiconductor traveling-wave amplifiers," IEEE Photon. Technol. Lett., vol. 6, pp. 50-52, 1994.

[8] M. C. Tatham, G. Sherlock, and L. D. Westbrook, "Compensation of fiber chromatic dispersion by optical-phase conjugation in a semiconductor-laser amplifier," Electron. Lett., vol. 29, pp. 1851-1852, 1993.

[9] G. P. Agrawal, "Highly nondegenerate four-wave mixing in semiconductor lasers due to spectral hole burning," Appl. Phys. Lett., vol. 51 , pp. 302-304, 1987.

[10] K. L. Hall, J. Mark, E. P. Ippen, and G. Eisenstein, "Femtosecond gain dynamics in InGaAsP optical amplifiers," Appl. Phys. Lett, vol. 56, pp. $1740-1742,1990$.

[11] R. Paiella and K. J. Vahala, "Highly nondegenerate four-wave mixing efficiency of an asymmetric coupled quantum-well structure," Appl. Phys. Lett., vol. 66, pp. 2619-2621, 1995. 
[12] S. Luryi, "Polarization oscillations in coupled quantum wells $A$ scheme for the generation of submillimeter electromagnetic waves," IEEE J. Quantum Electron., vol. 27, pp. 54-60, 1991.

[13] K. Leo, J. Shah, T. C. Damen, A. Schulze, T. Meier, S. Schmitt-Rink, P. Thomas, E. O. Göbel, S. L. Chuang, M. S. C. Luo, W. Schäfer, K. Köhler, and P. Ganser, "Dissipative dynamics of an electronic wave packet in a semiconductor double well potential," IEEE J. Quantum Electron., vol. 28, pp. 2498-2507, 1992.

[14] K. Leo, J. Shah, E. O. Göbel, T. C. Damen, S. Schmitt-Rink, W. Schäfer, and K. Köhler, "Coherent oscillations of a wave packet in a semiconductor double-quantum-well structure," Phys. Rev. Lett., vol. 66, pp. 201-204, 1991

[15] H. G. Roskos, M. C. Nuss, J. Shah, K. Leo, D. A. B. Miller, A. M. Fox, S. Schmitt-Rink, and K. Köhler, "Coherent submillimeter-wave emission from charge oscillations in a double-well potential," Phys. Rev. Lett., vol. 68, pp. 2216-2219, 1992.

[16] J. Thou, N. Park, K. Vahala, M. A. Newkirk, and B. I. Miller, "Study of interwell carrier transport by terahertz four-wave mixing in an optical amplifier with tensile and compressively strained quantum wells," $\mathrm{Appl}$. Phys. Lett., vol. 65, pp. 1897-1899, 1994.

[17] A. Uskov, J. Mørk, and J. Mark, "Wave mixing in semiconductor laser amplifiers due to carrier heating and spectral-hole burning," IEEE Ouantum Electron., vol. 30, pp. 1769-1781, 1994.

[18] K. Meissner, B. Fluegel, H. Gieben, G. Mohs, R. Binder, S. W. Koch and N. Peyghambarian, "Carrier dephasing in the gain region of an inverted semiconductor," Phys. Rev. B, vol. 50, pp. 17647-17650, 1994.

[19] J. Khurgin, "Second-order intersubband nonlinear-optical susceptibilities of asymmetric quantum-well structures," J. Opt. Soc. Am. B, vol. 6, pp. $1673-1682,1989$.
[20] M. Helm, in Intersubband Transitions in Ouantum Wells, E. Rosencher, B. Vinter, and B. Levine, Eds. Plenum: New York, 1992, p. 151.

[21] A. N. Korotkov, D. V. Averin, and K. K. Likharev, "TASERs: possible dc pumped terahertz lasers using interwell transitions in semiconductor heterostructures," Appl. Phys. Lett., vol. 65, pp. 1865-1867, 1994.

[22] C. Sirtori, F. Capasso, J. Faist, L. N. Pfeiffer, and K. W. West, "Farinfrared generation by doubly resonant difference frequency mixing in a coupled quantum-well two-dimensional electron gas system," Appl. Phys. Lett., vol. 65, pp. 445-447, 1994.

[23] E. R. Brown, K. A. Mcintosh, K. B. Nichols, and C. L. Dennis, "Photomixing up to $3.8 \mathrm{THz}$ in low-temperature-grown GaAs," Appl. Phys. Lett., vol. 66, pp. 285-287, 1995.

[24] B. N. Saif, B. D. Seery, J. Khurgin, and C. Wood, "Generation of 2.5 terahertz radiation by difference frequency mixing in $\mathrm{GaAs} / \mathrm{GaAlAs}$ multiple quantum-well waveguides," QELS, paper QthA6, 1995.

Roberto Paiella, photograph and biography not available at the time of publication.

Kerry J. Vahala (S'82-M'84), photograph and biography not available at the time of publication. 